\title{
Widespread activation of the DNA damage response in human pancreatic intraepithelial neoplasia
}

\author{
Jan-Bart M Koorstra ${ }^{1,2,4}$, Seung-Mo Hong ${ }^{1,4}$, Chanjuan Shi ${ }^{1}$, Alan K Meeker ${ }^{1}$, Ji Kon Ryu ${ }^{1}$, \\ George Johan A Offerhaus ${ }^{2}$, Michael G Goggins ${ }^{1,3}$, Ralph H Hruban ${ }^{1,3}$ and Anirban Maitra ${ }^{1,3,4}$ \\ ${ }^{1}$ Department of Pathology, The Sol Goldman Pancreatic Cancer Research Center, Johns Hopkins University \\ School of Medicine, Baltimore, MD, USA; ${ }^{2}$ Department of Pathology, University Medical Center, Utrecht, The \\ Netherlands; ${ }^{3}$ Department of Oncology, The Sol Goldman Pancreatic Cancer Research Center, Johns Hopkins \\ University School of Medicine, Baltimore, MD, USA and ${ }^{4}$ McKusick-Nathans Institute of Genetic Medicine, \\ Johns Hopkins University School of Medicine, Baltimore, MD, USA
}

Pancreatic intraepithelial neoplasia (PanIN) lesions are the most common non-invasive precursors of pancreatic adenocarcinoma. We postulated that accumulating DNA damage within the PanIN epithelium activates checkpoint mechanisms. Tissue microarrays were constructed from 81 surgically resected primary pancreatic adenocarcinomas and an independent set of 58 PanIN lesions (31 PanIN-1, 14 PanIN-2, and 13 PanIN3). Immunohistochemical labeling was carried out using anti- $\gamma \mathrm{H}_{2} \mathrm{AX} \mathrm{X}^{\mathrm{Ser} 139}$, anti-phosphoATM ${ }^{\mathrm{Ser} 1981}$, antiphosphoChk2 ${ }^{\text {Thr68 }}$, and anti-p53. A 'histologic score' combining area and intensity of labeling in the nuclear compartment was determined for each lesion. A progressive increase in $\gamma \mathrm{H} 2 \mathrm{AX} \mathrm{X}^{\mathrm{Ser} 139}$ labeling, consistent with escalating DNA damage, was observed in the non-invasive precursor lesions (scores of 4.34, 6.21, and 7.50, respectively, for PanIN-1, -2, and -3), compared with the pancreatic ductal epithelium (score 2.36) (ANOVA, $P<0.0001$ ). In conjunction, activation of the ataxia telangiectasia mutated (ATM)-Chk2 checkpoint pathway was observed in all histological grades of PanIN lesions. Specifically, PATM ${ }^{\text {Ser1981 }}$ histologic scores for PanIN-1, PanIN-2, and PanIN-3 were 4.83, 5.14, and 7.17, respectively, versus 2.33 for the ductal epithelium (ANOVA, $P<0.0001$ ); the corresponding scores for $\mathrm{pChk2}^{\mathrm{Thr68}}$ were $5.43,7.64$, and 5.44 in PanINs-1, -2 , and -3 , respectively, versus 2.75 in the ductal epithelium (ANOVA, $P<0.0001$ ). In contrast, absent to minimal nuclear p53 was observed in the ductal epithelium, and in PanINs-1 and -2 (a histologic score of 0-1.86), with a significant upregulation (corresponding to mutational inactivation) seen only at the stage of PanIN-3 and invasive neoplasia (histologic scores of 4.00 and 4.22). Nuclear p53 accumulation in cancers was associated with attenuation of the ATM-Chk2 checkpoint and a restitution to 'baseline' levels. To conclude, activation of the ATM-Chk2 checkpoint pathway is commonly observed in PanINs, likely in response to the accumulating DNA damage from events such as oncogene mutations and telomere dysfunction. Loss of p53 function appears to be a critical determinant for bypassing this checkpoint and the subsequent progression to invasive adenocarcinoma.

Modern Pathology (2009) 22, 1439-1445; doi:10.1038/modpathol.2009.114; published online 7 August 2009

Keywords: pancreatic cancer; pancreatic intraepithelial neoplasia; DNA damage; ATM; Chk2

\footnotetext{
*Correspondence: Dr A Maitra or S-M Hong, The Sol Goldman Pancreatic Cancer Research Center, Johns Hopkins University, 1550 Orleans Street, Room 345, Baltimore, 21231, MD, USA. E-mail: amaitra1@jhmi.edu or shong28@jhmi.edu

${ }^{4} \mathrm{~J}-\mathrm{BMK}$ and S-MH contributed equally to this paper.

Received 26 May 2009; revised 23 June 2009; accepted 26 June 2009; published online 7 August 2009
}

Adenocarcinoma of the pancreas affects approximately 38000 individuals each year in the United States of America, and nearly all patients die within months of diagnosis. ${ }^{1}$ A multistep model has recently been proposed for pancreatic adenocarcinomas, in which non-invasive precursor lesions in the pancreatic ducts undergo histological and genetic progression toward invasive cancer. ${ }^{2,3}$ These 
morphologically distinct non-invasive lesions have been classified under a uniform nomenclature scheme termed Pancreatic Intraepithelial Neoplasia (PanIN). We and others have shown that PanINs share many of the genetic aberrations associated with invasive adenocarcinomas, underscoring their classification as 'neoplasms' rather than a reactive/ hyperplastic process. ${ }^{4-10}$ Some of the genetic alterations are nearly ubiquitous (eg, oncogenic KRAS2 mutations and telomere dysfunction), ${ }^{4,5}$ suggesting that these are early events in the ductal epithelium, whereas others such as loss-of-function of the tumor suppressor gene, BRCA2, or upregulation of the GPIanchored protein, mesothelin, occur only in the most advanced PanIN lesions that precede invasive cancer. ${ }^{7,10}$

Autopsy studies have confirmed that PanIN lesions are, surprisingly, common in the general population, with more than $50 \%$ of the general population over 60 years harboring one or more lowgrade lesions in their pancreata. ${ }^{11}$ Nevertheless, despite this remarkably high prevalence and the presence of clonal genetic alterations, ${ }^{12}$ the overwhelming majority of low-grade PanINs do not progress to invasive adenocarcinoma, as obviously gauged by the annual incidence rates for this disease. One can speculate, therefore, that these low-grade PanINs either undergo apoptosis and are 'shed' from the body, or that intracellular checkpoint mechanisms come into effect, forestalling or entirely preventing their progression to higher-grade PanIN lesions (carcinoma in situ) and invasive cancer. Recent seminal studies have identified the DNA damage repair protein ataxia telangiectasia mutated (ATM) and its downstream target, the human homolog of the bacterial checkpoint, Chk2, as a pervasive checkpoint in human epithelial precancerous lesions. ${ }^{13-15}$ A variety of inciting factors, such as telomere dysfunction and oncogene-induced 'replication stress' can cause DNA damage in pre-cancerous lesions, activating the ATM-Chk2 checkpoint, thereby impeding their progression to invasive malignancy. ${ }^{16,17}$

We hypothesized that activation of the DNA damage response (DDR) checkpoint in the most common non-invasive precursor lesions of pancreatic adenocarcinoma could provide a putative explanation for the lack in connection between PanIN prevalence in the general population and the incidence of invasive adenocarcinoma. Herein, we confirm that activation of the ATM-Chk2 checkpoint is widespread in human PanIN lesions, including in the lowest grade (PanIN-1) lesions. This phenomenon appears to be a consequence of DNA replication stress and the occurrence of double-strand breaks, as measured by the progressive accumulation of phospho-histone $\gamma \mathrm{H} 2 \mathrm{AX}$, which forms a scaffold at double-strand breaks. ${ }^{18}$ We also provide evidence that loss of p53 function is a critical threshold event in the multistep progression of pancreatic cancer, occurring mostly at or beyond the stage of PanIN-3, allowing the neoplastic epithelium to bypass DDR-induced checkpoints and progress unimpeded into invasive adenocarcinoma.

\section{Materials and methods}

Tissue microarrays were prepared from the archival formalin-fixed paraffin-embedded sections of 81 surgically resected primary pancreatic adenocarcinomas, as previously described; ${ }^{19,20}$ this 'cancer tissue microarray' also included 73 cores of the non-neoplastic pancreatic ductal epithelium. An independent set of 58 PanIN lesions (31 PanIN-1, 14 PanIN-2, and 13 PanIN-3) was also arrayed on a 'PanIN tissue microarray', as previously described. ${ }^{4,7}$ For tissue microarray construction, representative areas containing morphologically defined cancers or PanINs were circled on the glass slides and used as a template. The tissue microarrays constructed using a manual Tissue Puncher/Arrayer (Beecher Instruments, Silver Spring, MD, USA) and a $1.4 \mathrm{~mm}$ core was punched from the donor block to ensure that adequate lesional tissue could be incorporated into the spot.

Immunohistochemistry was carried out as previously described. ${ }^{7}$ Briefly, unstained $5-\mu \mathrm{m}$ sections were cut from the paraffin block selected and deparaffinized by routine techniques. Thereafter, the sections were quenched with $3 \% \mathrm{H}_{2} \mathrm{O}_{2}$ for $10 \mathrm{~min}$. The slides were steamed in $10 \mathrm{mM}$ citrate buffer (ph 6.0) to unmask the epitopes for $20 \mathrm{~min}$ at $95^{\circ} \mathrm{C}$, and then allowed to cool down for $20 \mathrm{~min}$ to room temperature. Before incubating with the primary antibody, the slides were blocked for 30 min with a $10 \%$ fetal bovine serum solution (Invitrogen, Carlsbad, CA, USA). The following primary antibodies were used for this study: antiphospho $\gamma \mathrm{H} 2 \mathrm{AX}^{\mathrm{Ser} 139}$ (Upstate-Millipore, Millerica, MA, USA; dilution 1:200), anti-phosphoATM ${ }^{\text {Ser1981 }}$ (Rockland Immunochemicals, Boyertown, PA, USA; dilution 1:100), anti-phosphoChk2 ${ }^{\text {Thr68 }}$ (Cell Signaling Technology, Beverly, MA, USA; dilution 1:100), and anti-p53 (Santa Cruz Biotechnology, Santa Cruz, CA, USA; dilution 1:200). The specific phospho antibodies for $\gamma \mathrm{H} 2 \mathrm{AX}$, ATM, and Chk2 were selected based on the published association of phosphorylation at these sites with functional status of the respective protein. ${ }^{13,14,17,21}$ Labeling was detected with the the PowerVision + Poly-HRP IHC kit (Immunovision Technologies, Norwell, MA, USA) following the standard protocol. Slides were counterstained with Harris's hematoxyline solution. Negative controls (primary antibody replaced by serum from appropriate species) were used for each antibody in each run.

Immunohistochemical labeling was scored using a previously described histologic score (also known as HistoScore) scheme ${ }^{6,22,23}$ that takes into consideration both the area and intensity of labeling in the appropriate (nuclear) compartment. Specifically, 
intensity of labeling was designated as $0-3$ for absent, weak, moderate, and strong, and area of labeling was designated as $0-3$ for $<5 \%, 5-25 \%$, $26-50 \%$ and $>50 \%$, respectively. The lesional histologic score was calculated by the product of area and intensity, and subsequently, the average histologic scores for the individual histological grades of PanIN lesions, adenocarcinomas, and normal ductal epithelium were determined.

Statistical analyses were carried out using SPSS v17.0 (SPSS Inc., Chicago, IL, USA). Differential expressions of phospho- $\gamma \mathrm{H} 2 \mathrm{AX}^{\mathrm{Ser} 139}$, phosphoATM $^{\text {Ser1981 }}$, phospho-Chk2 ${ }^{\text {Thr68 }}$, and p53 proteins in normal pancreatic ductal epithelium, various grades of PanINs, and pancreatic ductal adenocarcinomas were compared by ANOVA and Duncan's multiple range tests. A $P<0.05$ was considered statistically significant.

\section{Results}

The mean histologic scores of phospho- $\gamma \mathrm{H} 2 \mathrm{AX}{ }^{\text {Ser139, }}$ phospho-ATM ${ }^{\text {Ser1981 }}$, phospho-Chk2 ${ }^{\text {Thr68 }}$, and p53 are summarized in Table 1a, whereas a graphical representation is provided in Figure 1. Statistically significant differences between histologic scores for PanINs-1, -2, -3 , or adenocarcinoma and that observed in non-neoplastic ductal epithelium for each of the four proteins are indicated in Table $1 \mathrm{~b}$ (calculated using Duncan's multiple range test, level of significance at $P<0.05$ ).

A progressive increase in phospho- $\gamma \mathrm{H} 2 \mathrm{AX}^{\mathrm{Ser139}}$ labeling, consistent with escalating DNA damage, was observed in PanIN lesions (histologic scores of
$4.34,6.21$, and 7.50, respectively for PanIN-1, -2, and -3), compared with ductal epithelium (histologic score 2.36) (ANOVA, $P<0.0001$ ). It is interesting to note that although invasive cancers had a significantly higher phospho- $\gamma \mathrm{H} 2 \mathrm{AX}^{\mathrm{Ser} 139}$ histologic score (4.53) than non-neoplastic ductal epithelium $(P<0.05$, Duncan's test $)$, it was significantly lower than that observed in both PanINs-2 and -3 , respectively. In conjunction with escalating double-strand breaks, a progressive activation of the ATM-Chk2 checkpoint was observed along the histological continuum of PanIN lesions. Specifically, phospho-ATM ${ }^{\text {Ser1981 }}$ histologic scores for PanIN-1, PanIN-2, and PanIN-3 were 4.83, 5.14, and 7.17, respectively, versus 2.33 for ductal epithelium (ANOVA, $P<0.0001$ ); the corresponding histologic scores for phospho-Chk2 ${ }^{\text {Thr68 }}$ were $5.43,7.64$, and 5.44 in PanINs-1, -2, and -3, respectively, versus 2.75 in ductal epithelium (ANOVA, $P<0.0001$ ). As indicated in Table $1 \mathrm{~b}$, the histologic score for each histological grade of PanIN was significantly higher than the corresponding histologic score in the ductal epithelium, for both proteins $(P<0.05$, Duncan's test). In both instances, attenuation of the checkpoint was observed in invasive cancers (histologic scores of 4.84 and 2.43, respectively, for phospho$\mathrm{ATM}^{\text {Ser1981 }}$ and phospho-Chk2 ${ }^{\text {Thr68}}$ ), such that in the case of phospho-Chk2 ${ }^{\text {Thr68 }}$ no significant difference in histologic scores was observed between cancer and the ductal epithelium. In contrast to the aforementioned proteins, absent to minimal nuclear p53 was observed in the ductal epithelium, as well as in PanINs-1 and 2 (histologic score of $0-1.86$ ), with a significant upregulation (corresponding to mutational inactivation) seen only at the stage of

Table 1a Summary of histologic scores for DDR markers in normal ductal epithelium, PanINs, and pancreatic ductal adenocarcinomas

\begin{tabular}{|c|c|c|c|c|c|c|}
\hline Antibody & $\begin{array}{l}\text { Normal duct } \\
\quad(\mathrm{N}=73)\end{array}$ & $\begin{array}{l}\text { PanIN-1 } \\
(\mathrm{N}=31)\end{array}$ & $\begin{array}{l}\text { PanIN-2 } \\
(\mathrm{N}=14)\end{array}$ & $\begin{array}{l}\text { PanIN-3 } \\
(\mathrm{N}=13)\end{array}$ & $\begin{array}{l}\text { Adenocarcinoma } \\
\qquad(\mathrm{N}=81)\end{array}$ & $\begin{array}{l}A N O V A \\
P \text {-value }\end{array}$ \\
\hline $\mathrm{p} \gamma \mathrm{H} 2 \mathrm{AX} \mathrm{X}^{\mathrm{Ser} 139}$ & 2.36 & 4.34 & 6.21 & 7.50 & 4.53 & $<0.0001^{\mathrm{a}}$ \\
\hline pATM ${ }^{\text {Ser1981 }}$ & 2.33 & 4.83 & 5.14 & 7.17 & 4.84 & $<0.0001^{\mathrm{a}}$ \\
\hline pChk2 $2^{\text {Thr68 }}$ & 2.75 & 5.43 & 7.64 & 5.44 & 2.43 & $<0.0001^{\mathrm{a}}$ \\
\hline p53 & 0 & 1.41 & 1.86 & 4.00 & 4.22 & $<0.0001^{\mathrm{a}}$ \\
\hline
\end{tabular}

DDR, DNA damage response and PanIN, pancreatic intraepithelial neoplasia.

${ }^{\mathrm{a}}$ Significant at the level of $P$-value $<0.05$.

Table 1b Statistically significant differences in histologic scores for DDR markers in PanINs and in adenocarcinomas, compared to histologic score in the non-neoplastic ductal epithelium

\begin{tabular}{lcccc}
\hline Antibody & $\begin{array}{c}\text { PanIN-1 } \\
(\mathrm{N}=31)\end{array}$ & $\begin{array}{c}\text { PanIN-2 } \\
(\mathrm{N}=14)\end{array}$ & $\begin{array}{c}\text { PanIN-3 } \\
(\mathrm{N}=13)\end{array}$ & $\begin{array}{c}\text { Adenocarcinoma } \\
(\mathrm{N}=81)\end{array}$ \\
\hline p H2AX $^{\text {Ser139 }}$ & $P<0.05^{\mathrm{a}}$ & $P<0.05^{\mathrm{a}}$ & $P<0.05^{\mathrm{a}}$ & $P<0.05^{\mathrm{a}}$ \\
pATM $^{\text {Ser1981 }}$ & $P<0.05^{\mathrm{a}}$ & $P<0.05^{\mathrm{a}}$ & $P<0.05^{\mathrm{a}}$ & $P<0.05^{\mathrm{a}}$ \\
pChk2 & $P<0.05^{\mathrm{a}}$ & $P<0.05^{\mathrm{a}}$ & $P<0.05^{\mathrm{a}}$ & $\mathrm{NS}$ \\
p53 & $\mathrm{NS}$ & $\mathrm{NS}$ & $P<0.05^{\mathrm{a}}$ & $P<0.05^{\mathrm{a}}$ \\
\hline
\end{tabular}

DDR, DNA damage response; NS, not significant and PanIN, pancreatic intraepithelial neoplasia.

${ }^{a}$ Significant at the level of $P$-value $<0.05$, Post-hoc Duncan's multiple range test. 

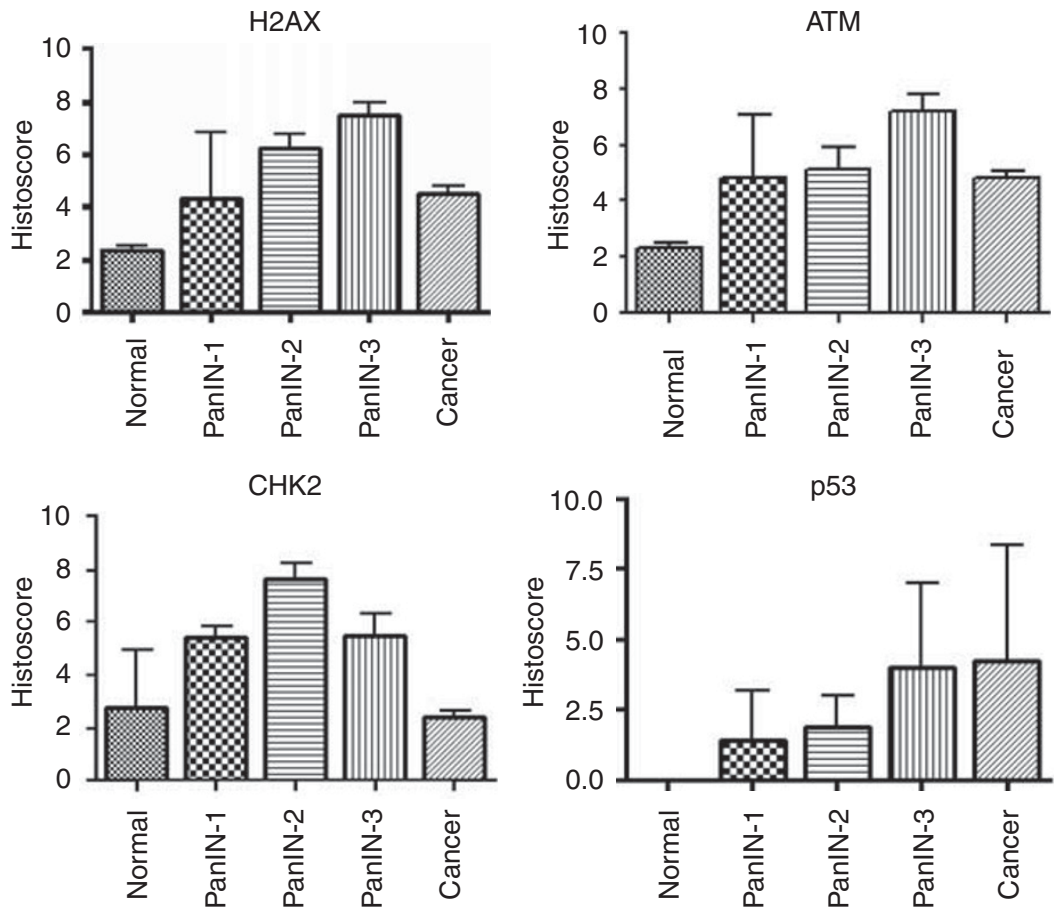

Figure 1 Histograms illustrating the histologic scores for each of the four proteins analyzed in this study, including phospho$\gamma \mathrm{H} 2 \mathrm{AX}{ }^{\text {Ser139 }}$, phospho-ataxia telangiectasia mutated ${ }^{\text {Ser1981 }}\left(\mathrm{ATM}^{\text {Ser1981 }}\right)$, phospho-Chk2 ${ }^{\text {Thr68 }}$, and p53. The histologic scores are stratified by the normal ductal epithelium, pancreatic intraepithelial neoplasias (PanINs)-1, -2, and -3, and invasive cancer. The mean histologic score and s.d. are represented for each grade of lesion. See text for details and statistical analyses.

PanIN-3 and invasive neoplasia (histologic scores of 4.00 and 4.22). Representative photomicrographs showing expression of these four proteins along the PanIN progression model culminating in invasive cancer are illustrated in Figure 2.

\section{Discussion}

A diverse array of intracellular signals may activate the so-called DDR checkpoint in cells, including DNA damage itself, as well as critical telomere shortening and oncogene activation (reviewed in ref. 24-28). Telomere dysfunction and oncogene activation appear to precipitate so-called 'replicative stress,' leading to DNA damage, and culminating in activation of the DDR checkpoint. ${ }^{16,17,25}$ The principal DNA damage phenotypes observed in the setting of the DDR are double-strand breaks, and these foci can be recognized by the binding of phosphorylated histone $\gamma \mathrm{H} 2 \mathrm{AX}$ to the damaged chromatin. ${ }^{18,29}$ The phosphorylated $\gamma \mathrm{H} 2 \mathrm{AX}$ forms a scaffold for the DNA repair machinery to engage at the site of doublestrand breaks, and therefore serves as a surrogate readout for DNA damage in cells. In mammalian cells, ATM, and its target, the bacterial checkpoint homolog protein Chk2, are the most important 'sensors' of double-strand breaks. ${ }^{28,30}$ Activation of ATM was originally described as an intracellular response to ionizing radiation, which, in turn, results in the activation of Chk2 protein through phosphorylation of a $\mathrm{Thr}^{68}$ moiety. ${ }^{31,32}$ As countless examples in experimental animal models and cognate human scenarios have documented, abrogation of the DDR checkpoint itself, or secondary defects in p53, enable cells to escape bypass this checkpoint even in the face of genomic damage (reviewed in refs. 27, 30, 33, 34).

In recent years, evidence has emerged to support aberrant activation of the DDR checkpoint in human epithelial pre-cancerous lesions. For example, Bartek et $\mathrm{al}^{14}$ described widespread abnormalities of the ATM-Chk2 axis in non-invasive precursor lesions of the human bladder, colon, and breast cancers, whereas Gorgoulis et $a l^{15}$ described comparable findings in the context of lung and epidermal tissues. In all of these instances, DDR checkpoint activation was accompanied by evidence of DNA double-strand breaks, as assessed by phosphorylated $\gamma \mathrm{H} 2 \mathrm{AX}$ expression. It was noted that p53 function was generally retained in the non-invasive precursor lesions, whereas progression to invasive cancer was accompanied by p53 inactivation, underscoring a selection pressure for clones with p53 dysfunction. ${ }^{14,15}$ Further, DDR in pre-cancerous lesions was observed before the onset of genomic instability that characterizes invasive cancer, suggesting that widespread allelic imbalances were not the underlying basis for checkpoint activation within the epithelium.

In this study, we report that histological progression along the PanIN continuum is associated with 

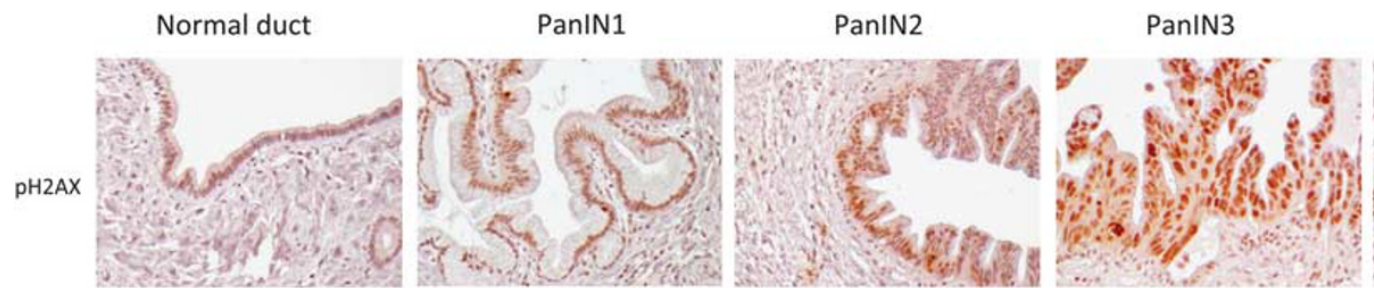

Adenocarcinoma
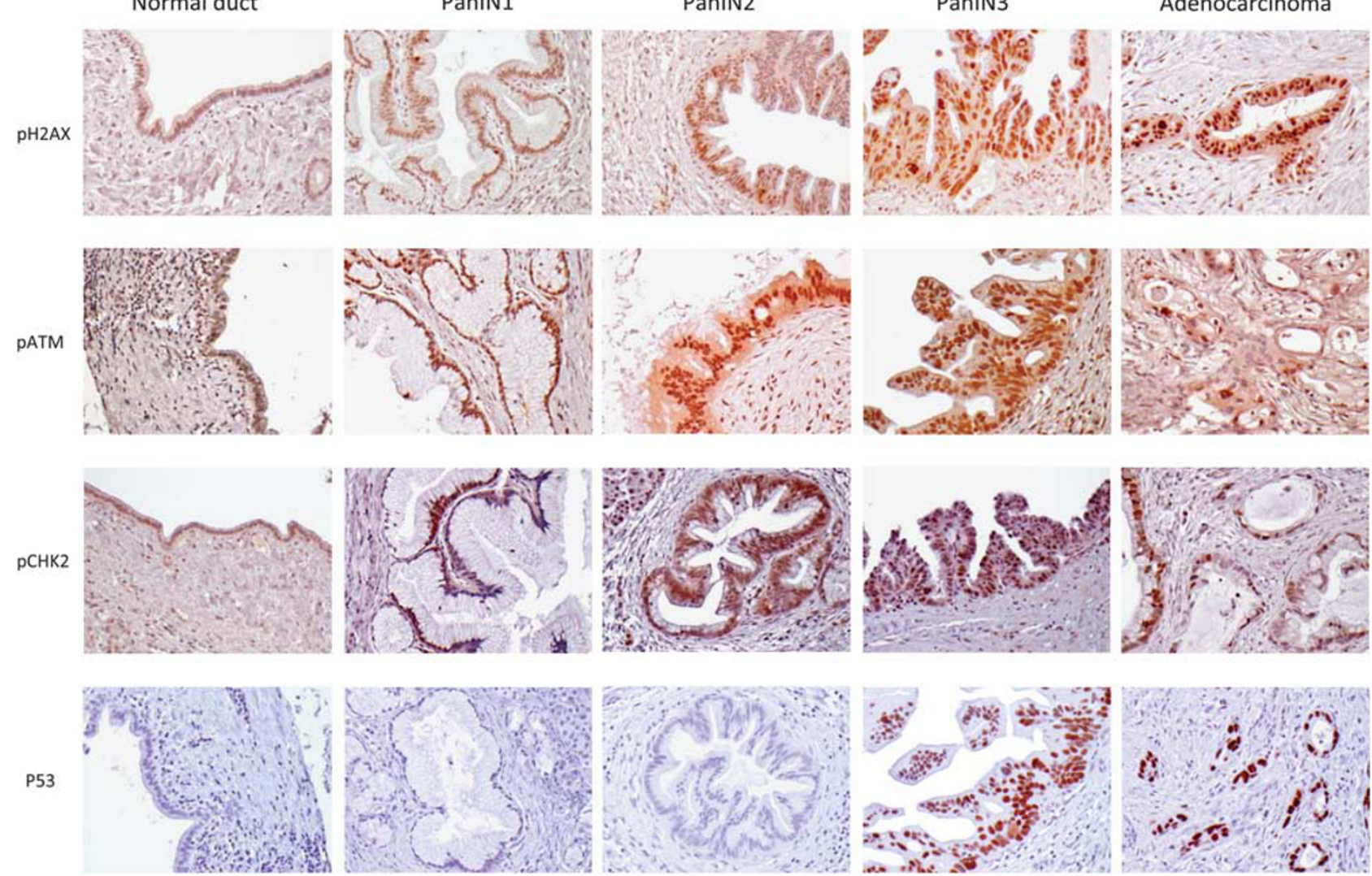

Figure 2 Representative photomicrographs illustrating the expression of phospho- $\gamma \mathrm{H} 2 \mathrm{AX}{ }^{\text {Ser139, }}$, phospho-ataxia telangiectasia mutated $^{\text {Ser1981 }}\left(\mathrm{ATM}^{\text {Ser1981 }}\right.$ ), phospho-Chk2 ${ }^{\text {Thr68 }}$, and p53 in various histological grades of the pancreatic ductal lesions.

an escalating degree of DNA damage, as assessed by phosphorylated $\gamma \mathrm{H} 2 \mathrm{AX}$ expression, as well as activation of the ATM-Chk2 checkpoint. For three of these proteins (phospho- $\gamma \mathrm{H} 2 \mathrm{AX}^{\mathrm{Ser} 139}$, phospho$\mathrm{ATM}^{\text {Ser1981, }}$, and phospho-Chk2 ${ }^{\text {Thr68 }}$ ), we found significant differences in the histological scores between non-neoplastic ductal epithelium and even PanIN-1, implying that DDR activation is one of the earliest molecular events in the multistep progression of pancreatic cancer. Nuclear accumulation of p53 is a reliable surrogate for mutational inactivation, ${ }^{35}$ and this was minimally observed up to the stage of PanIN-2, consistent with retained p53 function. In contrast, a significant upregulation of nuclear p53 was seen in PanIN-3 and in invasive cancer, reinforcing the need for loss of p53 function to bypass the DDR checkpoint. In our series, abrogation of p53 and progression to invasive adenocarcinoma was associated with a restitution of activated Chk2 expression to 'baseline' levels (ie, no significant differences in histologic scores between adenocarcinoma and non-neoplastic epithelium). Thus, the results described here are comparable to those observed in precursor lesions at other epithelial sites, ${ }^{14,15}$ and provide a unifying model for containing the unimpeded progression of precursor lesions to invasive cancer. A pictorial representation of these interdependent processes
(DNA damage, DDR, and p53 accumulation) along the PanIN histological continuum is presented in Figure 3, and underscores the temporal significance of p53 mutations in bypassing the ATM-Chk2 checkpoint.

One pertinent question that remains unanswered is the inciting event(s) leading to DDR within the pancreatic ducts, as genomic instability alone is unlikely to explain the rather widespread nature of the response. We believe that the reasons are multifactorial, with KRAS mutations and telomere dysfunction being the most likely culprits, as both are known to induce DNA damage. ${ }^{16,17,21,24,26,36}$ In fact, our group has previously shown that telomere attrition is present in $>90 \%$ of PanIN-1, ${ }^{4}$ providing a rational basis for 'replicative stress' and induction of DDR in the earliest precursor lesions. Re-activation of telomerase activity in invasive adenocarcinomas, as well as the consequent reduction in replicative stress, might underlie the paradoxical attenuation of double-strand breaks (ie, phospho$\gamma \mathrm{H}_{2} \mathrm{AX}^{\mathrm{Ser} 139}$ labeling) observed in the cancer samples, when compared with the levels in higher-grade PanIN lesions. The potential role of mutant KRAS in DDR has emerged from a recent mouse model of pancreatic cancer mediated by the expression of mutant Kras from its endogenous promoter, wherein markers attributable to senescence are observed in 


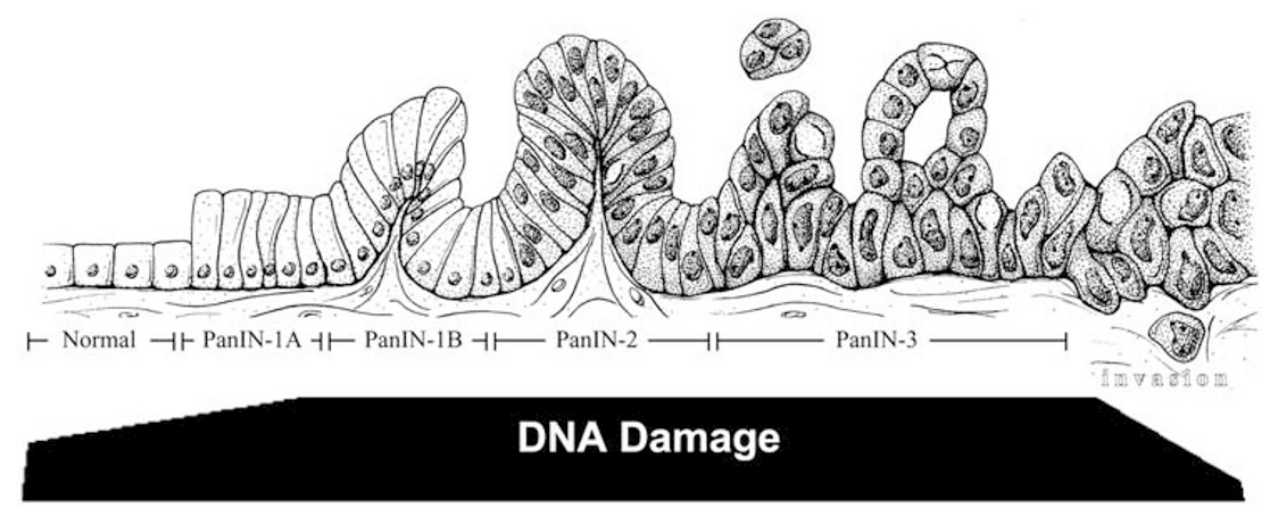

\section{DNA Damage response}

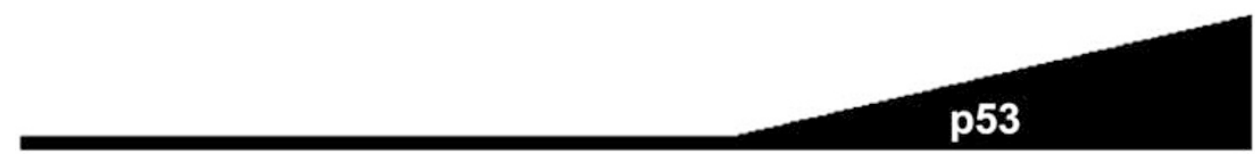

Figure 3 A proposed model of DNA damage response (DDR) mediated by the ataxia telangiectasia mutated (ATM)-Chk2 checkpoint in the pancreatic ductal epithelium. In response to double-strand breaks, DDR can be observed in the earliest pancreatic intraepithelial neoplasia (PanIN) lesions, and the increase in ATM-Chk2 expression parallels the histological progression to high-grade PanIN-3. Inactivation of p53 function at the stage of PanIN-3 and beyond is associated with bypass of the DDR checkpoint and progression to invasive cancer.

the murine PanIN lesions, but are lost on progression to invasive adenocarcinoma,. ${ }^{21}$ Furthering this parallel between human and murine disease is the observation that mice expressing mutant Kras alone develop invasive cancers in a minority of cases $(<10 \%),{ }^{37}$ while cooperating hits that allow cells to bypass checkpoints (eg, loss of Trp53 or Ink4a/Arf) result in complete and accelerated penetrance for the malignant phenotype. ${ }^{38-40}$

In summary, we report widespread activation of the DDR checkpoint in the most common non-invasive precursor lesions of pancreatic cancer, including in the lowest grade PanINs. We observe that a DDRinduced checkpoint in PanINs is contingent on retained p53 function, and that the inactivation of this 'gatekeeper' gene is likely one of the most critical events in opening the floodgates to invasive neoplasia. Finally, our results may provide a functional basis to the discordance between the rather common occurrence of PanIN lesions observed in the elderly population and the relatively uncommon incidence of pancreatic adenocarcinoma.

\section{Acknowledgements}

Supported by the Sol Goldman Pancreatic Cancer Research Center, the Michael Rolfe Foundation for Pancreatic Cancer Research and the NIH SPORE in GI Cancers P50CA062924. J-BMK is supported by the Netherlands Cancer Research Foundation (KWF) and International Exchange Program Grant provided by the University Medical Center Utrecht. The authors are grateful to Professor David A. Tuveson (Cambridge Research Institute, UK) for his insightful discussions on this paper.

\section{Disclosure/conflict of interest}

The authors declare no conflict of interest.

\section{References}

1 Maitra A, Hruban RH. Pancreatic cancer. Annu Rev Pathol 2008;3:157-188.

2 Hruban RH, Adsay NV, Albores-Saavedra J, et al. Pancreatic intraepithelial neoplasia: a new nomenclature and classification system for pancreatic duct lesions. Am J Surg Pathol 2001;25:579-586.

3 Hruban RH, Takaori K, Klimstra DS, et al. An illustrated consensus on the classification of pancreatic intraepithelial neoplasia and intraductal papillary mucinous neoplasms. Am J Surg Pathol 2004;28: 977-987.

4 van Heek NT, Meeker AK, Kern SE, et al. Telomere shortening is nearly universal in pancreatic intraepithelial neoplasia. Am J Pathol 2002;161:1541-1547.

5 Moskaluk CA, Hruban RH, Kern SE. p16 and K-ras gene mutations in the intraductal precursors of human pancreatic adenocarcinoma. Cancer Res 1997;57: 2140-2143. 
6 Maitra A, Ashfaq R, Gunn CR, et al. Cyclooxygenase 2 expression in pancreatic adenocarcinoma and pancreatic intraepithelial neoplasia: an immunohistochemical analysis with automated cellular imaging. Am J Clin Pathol 2002;118:194-201.

7 Maitra A, Adsay NV, Argani P, et al. Multicomponent analysis of the pancreatic adenocarcinoma progression model using a pancreatic intraepithelial neoplasia tissue microarray. Mod Pathol 2003;16:902-912.

8 Prasad NB, Biankin AV, Fukushima N, et al. Gene expression profiles in pancreatic intraepithelial neoplasia reflect the effects of Hedgehog signaling on pancreatic ductal epithelial cells. Cancer Res 2005;65:1619-1626.

9 Hustinx SR, Leoni LM, Yeo CJ, et al. Concordant loss of MTAP and p16/CDKN2A expression in pancreatic intraepithelial neoplasia: evidence of homozygous deletion in a noninvasive precursor lesion. Mod Pathol 2005;18:959-963.

10 Goggins M, Hruban RH, Kern SE. BRCA2 is inactivated late in the development of pancreatic intraepithelial neoplasia: evidence and implications. Am J Pathol 2000;156:1767-1771.

11 Cubilla AL, Fitzgerald PJ. Morphological lesions associated with human primary invasive nonendocrine pancreas cancer. Cancer Res 1976;36:2690-2698.

12 Koorstra JB, Feldmann G, Habbe N, et al. Morphogenesis of pancreatic cancer: role of pancreatic intraepithelial neoplasia (PanINs). Langenbecks Arch Surg 2008;393:561-570.

13 Bartkova J, Rezaei N, Liontos M, et al. Oncogeneinduced senescence is part of the tumorigenesis barrier imposed by DNA damage checkpoints. Nature 2006;444:633-637.

14 Bartkova J, Horejsi Z, Koed K, et al. DNA damage response as a candidate anti-cancer barrier in early human tumorigenesis. Nature 2005;434:864-870.

15 Gorgoulis VG, Vassiliou LV, Karakaidos P, et al. Activation of the DNA damage checkpoint and genomic instability in human precancerous lesions. Nature 2005;434:907-913.

16 Collado M, Blasco MA, Serrano M. Cellular senescence in cancer and aging. Cell 2007;130:223-233.

17 Di Micco R, Fumagalli M, Cicalese A, et al. Oncogeneinduced senescence is a DNA damage response triggered by DNA hyper-replication. Nature 2006;444:638-642.

18 Thiriet C, Hayes JJ. Chromatin in need of a fix: phosphorylation of $\mathrm{H} 2 \mathrm{AX}$ connects chromatin to DNA repair. Mol Cell 2005;18:617-622.

19 Salaria SN, Illei $\mathrm{P}$, Sharma $\mathrm{R}$, et al. Palladin is overexpressed in the non-neoplastic stroma of infiltrating ductal adenocarcinomas of the pancreas, but is only rarely overexpressed in neoplastic cells. Cancer Biol Ther 2007;6:324-328.

20 Cao D, Zhang Q, Wu LS, et al. Prognostic significance of maspin in pancreatic ductal adenocarcinoma: tissue microarray analysis of 223 surgically resected cases. Mod Pathol 2007;20:570-578.

21 Collado M, Gil J, Efeyan A, et al. Tumour biology: senescence in premalignant tumours. Nature 2005;436:642.
22 Hansel DE, Rahman A, Hermans J, et al. Liver metastases arising from well-differentiated pancreatic endocrine neoplasms demonstrate increased VEGF-C expression. Mod Pathol 2003;16:652-659.

$23 \mathrm{Fu}$ B, Luo M, Lakkur S, et al. Frequent genomic copy number gain and overexpression of GATA-6 in pancreatic carcinoma. Cancer Biol Ther 2008;7:1593-1601.

24 Sharpless NE, DePinho RA. Telomeres, stem cells, senescence, and cancer. J Clin Invest 2004;113:160-168.

25 Halazonetis TD, et al. An oncogene-induced DNA damage model for cancer development. Science 2008;319:1352-1355.

26 Mooi WJ, Peeper DS. Oncogene-induced cell senescence-halting on the road to cancer. N Engl J Med 2006;355:1037-1046.

27 Campisi J, d'Adda di Fagagna F. Cellular senescence: when bad things happen to good cells. Nat Rev Mol Cell Biol 2007;8:729-740.

28 Mallette FA, Ferbeyre G. The DNA damage signaling pathway connects oncogenic stress to cellular senescence. Cell Cycle 2007;6:1831-1836.

29 Sedelnikova OA, Horikawa I, Zimonjic DB, et al. Senescing human cells and ageing mice accumulate DNA lesions with unrepairable double-strand breaks. Nat Cell Biol 2004;6:168-170.

30 Bartek J, Bartkova J, Lukas J. DNA damage signalling guards against activated oncogenes and tumour progression. Oncogene 2007;26:7773-7779.

31 Matsuoka S, Huang M, Elledge SJ. Linkage of ATM to cell cycle regulation by the Chk2 protein kinase. Science 1998;282:1893-1897.

32 Ahn JY, Schwarz JK, Piwnica-Worms H, et al. Threonine 68 phosphorylation by ataxia telangiectasia mutated is required for efficient activation of Chk2 in response to ionizing radiation. Cancer Res 2000;60:5934-5936.

33 Kastan MB, Bartek J. Cell-cycle checkpoints and cancer. Nature 2004;432:316-323.

34 Vogelstein B, Kinzler KW. Cancer genes and the pathways they control. Nat Med 2004;10:789-799.

35 Baas IO, Mulder JW, Offerhaus GJ, et al. An evaluation of six antibodies for immunohistochemistry of mutant p53 gene product in archival colorectal neoplasms. J Pathol 1994;172:5-12.

36 Bardeesy N, Sharpless NE. RAS unplugged: negative feedback and oncogene-induced senescence. Cancer Cell 2006;10:451-453.

37 Hingorani SR, Petricoin EF, Maitra A, et al. Preinvasive and invasive ductal pancreatic cancer and its early detection in the mouse. Cancer Cell 2003;4:437-450.

38 Hingorani SR, Wang L, Multani AS, et al. Trp53R172H and KrasG12D cooperate to promote chromosomal instability and widely metastatic pancreatic ductal adenocarcinoma in mice. Cancer Cell 2005;7:469-483.

39 Aguirre AJ, Bardeesy N, Sinha M, et al. Activated Kras and Ink4a/Arf deficiency cooperate to produce metastatic pancreatic ductal adenocarcinoma. Genes Dev 2003;17:3112-3126.

40 Bardeesy N, Aguirre AJ, Chu GC, et al. Both p16(Ink4a) and the p19(Arf)-p53 pathway constrain progression of pancreatic adenocarcinoma in the mouse. Proc Natl Acad Sci USA 2006;103:5947-5952. 